Folia Histórica del Nordeste, $\mathbf{N}^{\circ} 19$ (Resistencia, 2011) IIGHI, IH - CONICET, UNNE

\title{
EPIDEMIOLOGÍA, SUBJETIVIDAD Y SALUD AMBIENTAL
}

\author{
Epidemiology, Subjectivity and Environmental Health
}

Rafael Antonio Carreras

\section{Resumen}

El artículo pretende contribuir al mejoramiento de los sistemas de información de salud ambiental interpretando el perfil epidemiológico de las comunidades a partir del análisis de la subjetividad y sus modos de producción desde enfoques complejos.

Los aportes de las ciencias positivistas han sido válidos para el estudio de la salud pero han mostrado límites para pensar la emancipación de distintas culturas vinculadas a derechos omitiendo procesos inherentes a los sujetos. Por lo tanto, las implicancias son muchas y requieren un gran esfuerzo para cuestionar las hipótesis que solidificaron determinadas disciplinas y generar una apertura reflexiva y crítica hacia un campo de problemas que propone más incertidumbres que solidez empírica - teórica: la subjetividad. En consecuencia, el escrito plantea comprender la subjetividad desde la consideración de contextos de cambios, las instituciones y sus vicisitudes, el reconocimiento por parte del estudio de la familia como unidad de análisis, incluyendo un análisis desde el pensamiento complejo.

$$
<\text { Epidemiología }><\text { Salud ambiental }><\text { Subjetividad }><\text { Instituciones }>
$$

\begin{abstract}
This article attempts to contribute to the improvement of the environmental health information systems through the interpretation of the epidemiologic profile of the communities from the analysis of the subjectivity and its modes of production from complex approaches.

The positivist sciences contribution has been valid for health research, but it has shown limitations to think of the emancipation of different cultures by the omission of processes inherent to the subjects. Therefore, the implications are many, and they require a great effort not only to challenge the hypothesis consolidated by certain disciplines, but also to generate reflexive and critical insights to Subjectivity. Consequently, Subjectivity is described, in this article, taking into consideration the contexts of change, the institutions and their vicissitudes, and the recognition of the family as unit of analysis. Besides, reflections about this topic, from the complex thought approach, are provided.
\end{abstract}

$$
<\text { Epidemiology }><\text { Environmental Health }><\text { Subjectivity }><\text { Institutions }>
$$




\section{Introducción}

La investigación pretende contribuir al mejoramiento de los sistemas de información en salud ambiental a partir de la inclusión del análisis de la subjetividad y sus modos de producción en la conformación del perfil epidemiológico de las comunidades.

Lo expresado supone entender a la comunidad como protagonista de su propia cultura, promoviendo su pensamiento crítico y su capacidad de reflexionar en el marco de su vida cotidiana.

Para esto, es necesario situar a la Epidemiología en sus dimensiones éticas, metodológicas y políticas, las cuales se inscriben en un campo socio-históricamente determinado pasible de ser alterado, intervenido y transformado. Con este fin, se precisarán los conceptos de salud y subjetividad, los modos de subjetivación y la familia como unidad de análisis. Además, se incluirán reflexiones de la temática desde el enfoque del pensamiento complejo.

La consolidación de una determinada perspectiva para entender el proceso salud- enfermedad - atención, se traduce necesariamente en abordajes pertinentes, no invasivos, que promuevan procesos emancipatorios en cada cultura.

\section{Salud y subjetividad: modos de producción en contextos de cambio}

La subjetividad y sus modos de producción abarcan la salud ambiental, la vida cotidiana y la cultura. Desde este trabajo, se sostiene que el reconocimiento de una situación en salud no es necesariamente observable ni se inscribe en un simple registro pasivo en el sujeto, sino que conlleva a una "propuesta activa de interpretación e intervención práctica, derivadas de modelos simbólicos del sujeto, congruentes con el orden social actual al que se pertenece, y al cual no puede cuestionar, en tanto funciona como el fundamento originario desde el cual da sentido a su experiencia" (Samaja, 2004).

En coincidencia con esa postura, los modelos socioculturales de la epidemiología proponen que las fronteras entre salud y enfermedad son establecidas a partir de las experiencias de sufrimiento de cada cultura, según cómo éstas sean narradas, y qué rituales se instrumenten para dar respuesta a aquello que el sufrimiento deteriora.

Es importante subrayar que se hace referencia a la salud ambiental considerando que existe una relación insustituible con el medio natural en el que vive el sujeto y su devenir histórico.

En este sentido, referirse a la salud de un sujeto, implica un entramado psicosocial que inscribe al sujeto en su vida cotidiana, su cultura, en una dimensión relacional que le otorga existencia, interpela su subjetividad y se adentra en la perspectiva de su mundo construido, en su cosmovisión y en su irrenunciable historia y en sus condiciones concretas de existencia. 
A continuación presentaré un breve desarrollo explicativo de los conceptos considerados centrales para reconocer lo presentado:

a) El hábitat: Es el lugar en el cual se dan los primeros aprendizajes e interacciones sociales que permiten a la persona configurar su mundo interno, establecer lazos de pertenencia constitutivos de su subjetividad, y desarrollar el potencial sensorial, que le permite ubicarse geográfica y temporalmente. El hábitat otorga a la persona un modo de comunicarse esencial para la satisfacción de necesidades, desde la construcción de un "lenguaje de objetos producidos" (Samaja, 2004) necesarios para la constitución de una comunidad.

Por otra parte, algunos especialistas mencionan que la internalización de objetos, arquitecturas y paisajes del hábitat se inscribe en la identidad del sujeto, sólo, desde el sentido otorgado por el grupo social de pertenencia. Así, un árbol, podrá ser un lugar de descanso, objeto de intercambio para la subsistencia, un lugar de encuentro o todos a la vez. El hábitat y la salud están vinculadas entre sí y se autodeterminan, son parte de un mismo concepto.

El hábitat es un lugar de pertenencia que como tal refiere a una identidad, a un plus de sentidos ${ }^{1}$. Habitar, no es sólo desplazamiento de cuerpo y movimiento sino también un despliegue de sentidos y significados simbólicos que configura al mundo que rodea, es un afincamiento subjetivo, pues, esta operación previa posibilita construir aquello que es habitable.

Por lo tanto, el hábitat posee una función clave para la subsistencia y para reconocer, en el lenguaje de los sujetos, modos de formular preguntas para resolver la existencia que transcurre en la vida cotidiana ${ }^{2}$ supone un mundo en movimiento.

b) Vida cotidiana: La experiencia constituye una categoría central, al punto de subjetivar las prácticas sociales. Cada experiencia hace a la cotidianeidad de un sujeto, por lo tanto una sociedad constituye múltiples cotidianeidades de distintos sujetos. La cotidianeidad remite a una familiaridad, que muchas veces se presenta como incuestionable y acrítica, por el carácter natural, secuencial y repetitivo en que se despliega.

Uno de los aspectos más relevantes de la vida cotidiana es que "es centralmente el escenario de la re-producción social, está indisociablemente vinculada a lo que en un momento especifico y en una cultura particular se asume como legítimo, normal, necesario para garantizar la continuidad" (Reguillo, 2000)

\footnotetext{
1 "La dimensión plus está en todas partes: en relación a la arquitectura y otras disciplinas, entre el proyecto y el edificio, entre el espacio y el habitarlo, entre el objeto y la subjetividad." (Lewkowcz Sztulwark, 2003)

2 "La vida cotidiana es un conjunto multitudinario de hechos, de actos, objetos, relaciones y actividades que se nos presentan en forma dramática, es decir, como acción, como mundo-en-movimiento. Son hechos múltiples y heterogéneos, de difícil clasificación, en los que toma cuerpo y se patentiza en forma fragmentaria e inmediata la organización social de la relación entre necesidades y metas" (Quiroga, 1996)
} 
La subjetividad adquiere su máxima expresión en la vida cotidiana. Ésta es el lugar donde aquella se estructura y se desestructura, de allí su lugar central para pensarla o cuestionarla.

c) Subjetividad: Para Fernández (1997), la subjetividad puede ser pensada como "campo de problemas". Con esto refiere a la necesidad de conceptualizarla no sólo desde lo descriptivo, sino desde la inscripción en su devenir sociohistórico, en el cual su modo de producción es mucho más que las formas de existencia del sujeto: "Es histórica no sólo en el sentido que surge en un proceso, que es efecto de determinadas variables históricas, en el sentido de la historia social, que varía en las diferentes culturas y sufre transformaciones a partir de mutaciones que se dan en sistemas políticos históricos". (Bleichmar, 2007)

El sujeto y su territorio son productores de subjetividad. Ese territorio material y simbólico sólo posee existencia si se comparte y si se pertenece con otros. En este sentido, la subjetividad es experiencia, es praxis social, encuentros y desencuentros, resistencia y diversidad, orientados desde una invención colectiva: la sociedad y la cultura ${ }^{3}$ de pertenencia. Reguillo Cruz (2006), entiende a la subjetividad como:

"la compleja trama de los modos en que lo social se encarna en los cuerpos y otorga al individuo históricamente situado tanto la posibilidad de reproducción de ese orden social como las de su negación, impugnación y transformación; es el intento de hacer salir de la clandestinidad los «dispositivos de percepción y respuesta» con que los actores sociales enfrentan la incertidumbre y los riesgos epocales" (Reguillo, 2006)

También, la subjetividad es un modo de dar respuestas, de transformar, de volver rutina el sentido de la vida y de la muerte, desde la propia configuración, desde la propia organización de las secuencias vivenciales; es un lugar para resistir el sufrimiento, para develar la existencia placentera y repensar modos de hacer, tomas de decisiones hacia estilos de vida saludables. Esos modos de resistencia modelan la subjetividad de forma inestable y con la fuerza de confrontar el malestar, las miserias y las incertidumbres de la vida cotidiana en épocas de cambios.

\section{Modos de subjetivación: instituciones, contingencias y agenciamiento}

La institución y la contingencia son aspectos que se ponen en juego en la vida cotidiana, por lo que requieren ser explicitados al aproximarnos a los conceptos de

\footnotetext{
${ }^{3}$ En este trabajo, se entiende a la cultura como la "capacidad de comunicarse, de entenderse: la cultura supone modos compartidos de significar el mundo que proveen orientaciones hacia la acción, lo que implica que cuando se comparte la cultura los comportamientos de los demás se tornan inteligibles y relativamente predecibles. (Margulis, 2009)
} 
subjetividad, salud y hábitat. Consideraremos a la institución como producto de la tensión entre institucionalización y desinstitucionalización como signos de la época, y a la contingencia como condición esperable que caracteriza aspectos sociales, políticos e históricos y que a su vez está presente e incide en la antinomia mencionada.

La función primaria de la institución es la de promover la socialización, en ese marco es posible entender la sociedad como un conglomerado de instituciones que buscan al menos ser funcionales mutuamente en pos de un objetivo más amplio.

La socialización es un proceso en el cual se combinan las condiciones psicológicas, socioculturales y biológicas en un sujeto. Es el proceso a través del cual se incorporan habilidades, normas, valores, pautas y reglas para ser socialmente aceptado. Es un recurso más que la sociedad utiliza para que las personas adopten comportamientos comunes.

Es un movimiento que se da hacia el sujeto pero en el que éste también experimenta un proceso inverso que podríamos llamar habituación. Éste sugiere al ser humano estructurarse a partir de normas y pautas ritualizadas que le permiten anticiparse a la próxima actividad, restringiendo muchas veces las oportunidades.

El proceso de habituación, antecede a toda institucionalización "provee el rumbo y la especialización de la actividad humana que faltan en el equipo biológico del hombre, aliviando de esa manera la acumulación de tensiones resultante de los impulsos no dirigidos" (Berger y Luckmann, 1966). Según estos autores, dos procesos graduales se ponen en juego para la inserción social de las personas y su construcción de la realidad, según la perspectiva del interaccionismo simbólico: la Socialización primaria $^{4}$ y la secundaria ${ }^{5}$.

A continuación, se describirán dos procesos que se manifiestan en la relación entre subjetividad, institución y contexto, a modo de tensión:

El proceso de institucionalización, refiere a la descripción de nociones tales como subjetivación, agenciamiento y afiliación, fundamentales para su comprensión. Los modos de subjetivación son formas en las cuales las instituciones de la modernidad han hecho efectivos los sistemas de creencias, la trasmisión de cultura, los "mandatos pulsionales"6 (Samaja, 2004). La condición subjetivante de una institución hace alusión a la capacidad de adscripción y de afiliación que un sujeto dispone hacia las lógicas

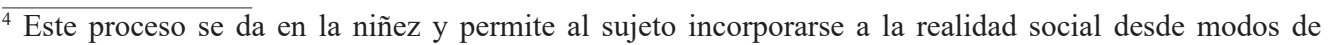
interacción y de relación social que le permiten construir vínculos con otros a partir de la satisfacción de necesidades.

${ }^{5}$ Este proceso incorpora al sujeto a la realidad institucional a partir del aprendizaje de roles. Si bien el inicio no depende de una edad evolutiva precisa este proceso comienza cuando se incorpora el "Otro generalizado", esto es, cuando se asumen los sistemas de significaciones socialmente compartidos en un proceso sincrético entre individuo y sociedad.

${ }^{6}$ Me refiero a este concepto, a los imperativos que impone cada cultura para su reproducción que se ponen en tensión con los procesos sociohistórico que atraviesa cada sociedad, el concepto de mandato no es más que exigencias culturales. Las pulsiones, son respuestas a esas exigencias que involucran las demandas sociales y familiares de cada época según el lugar que ocupe el sujeto en la estructura social, según la estructura de oportunidades disponible para ese sujeto y su unidad primaria.
} 
y reglas de juego. La institucionalización del sujeto, protege socialmente y posee implicancias identitarias, en tanto conlleva a un sentimiento de pertenencia, inscripto en un orden social sancionado. Esta pertenencia supone fortalecimientos de redes sociales, por tanto, un soporte socio-afectivo que incide en las relaciones de mutualidad y apoyo.

Las personas participan de las instituciones en tanto se reconocen en ellas e incorporan una disposición relacional que, a su vez, supone un enraizamiento institucional, un agenciamiento que atraviesa y modela la subjetividad.

Cabe remarcar que esta tríada institución/ agenciamientos ${ }^{7} /$ sujeto, guarda una relación con el contexto socio-histórico. Según Ana Quiroga (1996), en momentos en donde amenaza la exclusión social, se produce un deterioro en las relaciones sociales y ocurre un desapego institucional que atenta la cohesión social.

En relación a esto último, describiré el proceso de desinstitucionalización que sólo es comprensible en las condiciones sociales y los modos de organización actual. Dicho proceso posee un sentido bisémico a deconstruir:

En el primer sentido, el externalizar un sujeto de un instituto psiquiátrico reviste un carácter emancipatorio y hasta conlleva una discusión sobre los criterios de normalidad social y comportamientos aceptables (Castel, 1980; Samaja, 2004; Brehil, 2003). Aquí la desinstitucionalización constituye un escenario para potenciar avances en las sociedades, problematizando ${ }^{8}$ los acuerdos de sociabilidad, y las perspectivas hegemónicas de convivencia social.

En el segundo sentido, la desinstitucionalización es producto del desmantelamiento del Estado. Es decir, significa la pérdida de sentidos y guías de las instituciones que constituyen una sociedad, lo que produce una externalización del sujeto a modo de expulsión, en tanto su orden jurídico-material no lo protege sino que lo sitúa en modos de habitar la subjetividad a la intemperie, implicando una inscripción institucional debilitada, disminuyendo en el sujeto la capacidad de decidir, en la cual se pone de manifiesto la incertidumbre como sentimiento asociado.

Esta incertidumbre es pura contingencia, y abre paso a la "apertura necesaria de diferentes posibilidades de fijación de toda existencia" (Ema López, 2004). Como se dijo anteriormente, la subjetividad es sujeción al cambio, y este cambio o transformación se inscribe en un contexto socio-histórico particular, en el cual la cultura juega un papel muy importante, como productora y como producto.

Por lo descripto, retomando la tensión entre proceso de institucionalización y proceso de desinstitucionalización, es necesario hablar de modos de producción de subjetividad, en contextos institucionales, conceptualización que permite situar, o producir un anclaje en el sujeto, la historia y su cultura.

\footnotetext{
${ }^{7}$ Se entiende este concepto como "una multiplicidad que comporta muchos términos heterogéneos y que establece uniones, relaciones entre ellos [...] la única unidad del agenciamiento es de co-funcionamiento: una simbiosis, una simpatía". (Deleuze y Parnet, 1997, citado por Ema López, 2004).

${ }^{8}$ La problematización se entiende como un proceso critico de conocimiento en el cual se desecha el carácter natural relacionado con ciertos fenómenos, reflexionando sobre sus causas y sus consecuencias. (Montero, 2004).
} 
"Con la noción de producción de subjetividad aludimos a una subjetividad que no es sinónimo de sujeto psíquico, que no es meramente mental o discursiva sino que engloba las acciones y las prácticas, los cuerpos y sus intensidades; que se produce en él entre otros y que es, por tanto, un nudo de múltiples inscripciones deseantes, históricas, políticas, económicas, simbólicas, psíquicas, sexuales, etc. (Fernández, 2006).

Ahora bien, estos anclajes no se desarrollan al igual que en las sociedades de la modernidad, es por eso que una tensión entre institucionalización/ desinstitucionalización, debe incorporar la noción de afincamiento de territorios, para referir al movimiento en esta tensión, sea cual fuere la institución o el espacio de socialización en el cual la persona se desplace. Los afincamientos territoriales son aquellos espacios sociales que el sujeto habita, que poseen una eficacia simbólica y que provocan una inscripción subjetiva. Espacios que los sujetos eligen para erigirse como sujetos de transformación y resistencia y donde la contingencia circula con mucha transparencia y en forma reiterada. Es a través de estos afincamientos que los modos de producción de subjetividad adquieren su máxima expresión, en tanto inciden en el contexto social que los circunda, y su enunciación es performativa. Sólo así los agenciamientos son posibles. Es importante destacar que los agenciamientos producen enunciados colectivos, por lo tanto, el agenciamiento siempre es de un grupo de personas y no de un solo individuo, "son máquinas de expresión que desbordan el sujeto y el lenguaje (...) es un estado de mezcla de los cuerpos de una sociedad (sus atracciones, repulsiones, simpatías, rechazos, etc.) que afectan los cuerpos unos en relación con los otros, pero es una máquina social que excede todo objeto" (Fernández, 2007).

No obstante, las lógicas para pensar la salud, la subjetividad y los procesos de enunciación parecen recorrer estos conceptos, buscando los enunciados en su carácter performativo y transformador y reflexionando desde las acciones colectivas, deseantes y emancipatorias para una mayor eficacia. Las condiciones aleatorias en las que se presenta la vida cotidiana hoy y el modo en que emerge su carácter impredecible en el devenir subjetivo, propone múltiples formas de fijar la existencia, deja marcas que no necesariamente guardan relación unas con otras.

\section{La familia como unidad de análisis}

El lugar de la familia es vital y punto de partida para pensar a la comunidad y sus prácticas saludables. Por esto, es necesario situarla como unidad de análisis, repensando las configuraciones, lógicas y efectos subjetivantes.

Se reconoce que la familia inscribe en cada sujeto una matriz interna de aprendizaje, a partir de su participación en ella desde temprana edad. Esto permite al sujeto organizar y significar un sinnúmero de experiencias cotidianas, y lo provee de un conocimiento práctico que le permite operar e interactuar con el mundo que lo 
rodea. Se trata de una inscripción subjetiva de un modelo. "Esta matriz o modelo es una estructura compleja y contradictoria con una infraestructura biológica. Está socialmente determinada e incluye no sólo aspectos conceptuales, sino también emocionales, afectivos y esquemas de acción". (Quiroga, 1996).

Para la autora recién citada, estas matrices constituyen estructuras en movimiento, por la multiplicidad de experiencias a la que los sujetos están expuestos y por las que son susceptibles de ser modificados. Su carácter es complejo y abierto. La familia, predispone y subjetiva a quienes participan de ella, con intensidades distintas. Constituye en sí una matriz de aprendizaje y es una unidad primaria grupal, a la que la mayoría de los individuos adscriben en sus primeros años de vida.

También es importante reconocer su rol de agencia para socializar, porque el concepto de agencia ya ubica al grupo familiar como algo en constante desplazamiento $\mathrm{y}$ es uno de los elementos centrales que se quiere destacar.

La condición de agente para el sujeto implica situarlo en un contexto, en un orden social, tomando la relación contexto-familia. La condición de agencia implica vincular la capacidad de acción con una concepción relacional del poder de esa agencia, habla de responsabilidad, de su carácter de mediación de flujos entre la acción y el compromiso ético-político, de su capacidad para generar conexiones, encuentros entre distintos agentes. (Ema López, 2004).

Esa es la potencia de la familia pensada como unidad de análisis desde el concepto de agencia. Un nodo problemático de flujos que se conectan y desconectan fuertemente. La dimensión subjetiva de cada participante reviste en el encuentro con el otro, pasiones, sentimientos, amor y también malestar.

Como toda institución de la modernidad, la familia ha sufrido modificaciones a consecuencia de los cambios vertiginosos sociales, políticos y culturales de los últimos veinte años. Ahora bien, podríamos preguntarnos entonces ¿qué hace que personas se constituyan en una familia?

Las condiciones actuales de agrupamiento familiar son disposiciones que no necesariamente pueden estar reguladas por el lazo sanguíneo. Familia es el territorio compartido, es el hábitat de pertenencia y sus representaciones simbólicas, aquello que los agentes se representan como familiar, como propio, el "sentirse en casa", que supone reciprocidad sensorial y afectiva, un campo semántico compartido y una mutua representación interna de cada uno de los que participa. El hábitat y la pertenencia son atributos de una identidad familiar.

Todo miembro de la familia tiene diferentes roles, funciones y responsabilidades cuyas modificaciones son producto de los cambios sociales (Duschatzky y Corea, 2002; Jelin, 1998; Julien, 1993).

Algunos investigadores, se centran en el cambio de la figura paterna aseverando, por un lado, su destitución simbólica y una progresiva asunción de este rol por parte de grupos semejantes. Por ejemplo, son las mujeres-madres quienes resuelven muchas 
veces la supervivencia, lo cual trastoca la función tradicional "pasiva" de la mujer. A esto se suman la pérdida de la condición salarial de muchos hombres, el ingreso masivo de nuevas tecnologías que suplen la transferencia de conocimiento intergeneracional y el ejercicio de la paternidad de manera precoz.

Otros análisis de gran relevancia de los autores mencionados refieren a una progresiva sustitución del lugar del padre que procede de los grandes avances realizados por la medicina, por ejemplo: la inseminación artificial. A esto se suma que los discursos sociales cada vez sostienen menos el lugar del padre. Hay, en ese sentido, legislaciones que permiten la adopción de una mujer sin necesidad de que contraiga algún tipo de relación conyugal; por lo tanto, una mujer posee libertad de dar a luz sin un padre, ejercer la crianza, adoptar, etc.

Por consiguiente, lo que hace que personas se constituyan en una familia, puede ser pensado desde el hábitat de pertenencia y sobre todo, desde la experiencia, la historia y la cotidianeidad de las personas y, lo que resulta más relevante, desde la intensidad de los vínculos y su estructura relacional. En este sentido, las acciones destinadas a salud sólo pueden ser pensadas y diseñadas en el territorio y en una covisión de los distintos actores de una comunidad.

\section{Abordando la complejidad}

La subjetividad se expresa en las emociones, en los sentidos y significados que los sujetos le otorgan al mundo que los rodea, a cuya configuración es inherente la experiencia, las relaciones sociales y la participación en la cultura.

Historia, contexto, vida cotidiana, hábitat y salud constituyen fragmentos de un mismo texto. Por esto, la subjetividad es vista como un campo de problemas a deconstruir, en tanto sitúa un contexto socio-histórico y un territorio socialmente definido en permanentes mutaciones, en el cual las certezas se disponen a modo de fuerzas sin consistencia, por momentos antagónicas, por momentos difusas.

Se ha tratado de hacer un recorrido sobre algunas dimensiones constitutivas de la subjetividad, a los fines de entenderla como campo de problemas, y dar cuenta de la complejidad que implica su abordaje, mostrando cómo su comprensión requiere borrar fronteras disciplinares. Es decir, se necesita la participación de distintas disciplinas, problematizando su episteme hacia la producción de saberes transdisciplinarios ${ }^{9}$, en interlocución con los distintos saberes y modos de vida de una comunidad, a fin de recuperar y reconocer expresiones culturales, identitarias, diversas que distancian muchas veces a los grupos humanos.

\footnotetext{
${ }^{9}$ Con este concepto me refiero a un modo de abordar problemas más allá de la propia disciplina. Aquello que pueda problematizar un campo que no es propio, a enfrentar los impensables de cada saber disciplinario. Edgard Morin, nomina sistemas abiertos a aquellos fenómenos que no pueden ser pensados desde un solo cuerpo teórico. Implicando la posibilidad de transformación que incluye la historia, el devenir (Morin, 1994).
} 
Entonces, se afirma que la subjetividad y su modo de producción refieren a componentes del contexto social, la experiencia y la contingencia. Se sujeta eminentemente a la vida cotidiana y a los modos de vida, entendidos éstos como "la realidad histórica total que pertenece a un grupo social determinado... en cuanto es vivida en la praxis del grupo, en todo en cuanto identifica a sus miembros" (Brehil, 2003).

Desde una perspectiva epidemiológica emancipadora, abordar la salud en términos ambientales, implica por un lado reconocer los modos de producción de subjetividad, y por otro, provocar una ruptura con todas las ataduras materiales, políticas y culturales que ha creado la propia sociedad capitalista. Por lo tanto, es necesario remarcar el carácter transformador que implica el paradigma de la epidemiología, en tanto se funda en un cambio social, en formas de pensar la salud desde procesos que se ponen en juego y de fuerzas que no siempre muestran un correlato, pero que priorizan la mirada del sujeto sobre el medio que lo rodea.

La epidemiología crítica ha realizado numerosos esfuerzos y aportes desde una perspectiva de resistencia a modelos de salud consolidados, buscando desde sus bases teóricas y empíricas la superación de paradigmas dominantes. Por ello, la subjetividad y el proceso salud - enfermedad - atención, desde esta perspectiva teórica, deben ser pensados como praxis hacia la emancipación cultural.

El propósito de este escrito es abrir campos de interrogación sobre la subjetividad. Es por esto que, para su estudio, es necesario iniciar un proceso de desdiciplinamiento que "implica complejos procedimientos elucidatorios: desnaturalizar los dominios de objetos instituidos, deconstruir las lógicas desde donde han operado sus principios de ordenamiento, interrogar los a priori desde los que un campo de saberes y prácticas ha construido sus conceptualizaciones". (Fernández, 2007).

Esta propuesta resulta ambiciosa, y sugiere la posibilidad de desterritorializar los campos específicos de cada disciplina con el objeto de poder irrumpir o al menos problematizar las miradas unidisciplinarias que suelen construirse. Pero este largo camino echa por tierra la posibilidad de aplicar a la experiencia un corpus de conocimientos ya objetivado, porque justamente ahí se omitiría pensar desde la complejidad y desde la naturaleza del acontecimiento ${ }^{10}$.

\section{Consideraciones finales}

Para la epidemiología crítica y en particular la salud ambiental, es necesario incluir la subjetividad como un campo de problemas a elucidar, con el objeto de alcanzar una interpretación eficaz del proceso salud-enfermedad-atención.

Por consiguiente, es necesaria una perspectiva que recupere la percepción del sujeto y provoque determinadas rupturas con los paradigmas hegemónicos que no han

\footnotetext{
${ }^{10}$ Este concepto refiere a la praxis imprevista, aleatoria y contingente que pueda presentar el comportamiento de un fenómeno social.
} 
podido demostrar eficacia en la distribución equitativa de las condiciones de vida. Así, la familia se constituye en una institución clave para iniciar el análisis, reconociendo la potencia de su matriz primaria de aprendizaje y su incidencia en los sujetos modelando los modos de organizar las experiencias sucesivas y sus adscripciones culturales.

La puesta en práctica de estos supuestos implica pensar la salud hacia formas que reconozcan los procesos de habituación, vida cotidiana, y cultura en el marco de un momento sociohistórico particular situado.

La epidemiología crítica, como ciencia emancipadora, propone alternativas de poder/salud/comunidad en pos de una mejora de la calidad de vida de las personas, reconociendo la perspectiva de los agentes comunitarios.

Finalmente, es indispensable destacar que la complejidad expuesta supone el saber popular y el saber científico, en una relación dialéctica y no dicotómica, como signos claves para pensar y formular diseños y abordajes eficaces en el proceso salud/ enfermedad/atención hacia un monitoreo epidemiológico comunitario.

\section{Referencias Bibliográficas}

Almeida Filho de Naomar y María Zélia Rouquayrol. 2008. Introducción a la Epidemiología. Buenos Aires, Lugar.

Almeida Filho de Naomar. 2000. La Ciencia tímida. Ensayo de deconstrucción de la Epidemiología. Buenos Aires, Lugar.

Berger, Peter Ludwig y Thomas Luckmann. 1966. La construcción social de la realidad. Buenos Aires, Amorrortu.

Bleichmar, Silvia. 2007. La subjetividad en riesgo. Buenos Aires, Topia.

Brehil, Jaime. 2003. Epidemiología crítica. Ciencia emancipadora e interculturalidad. Buenos Aires, Lugar.

Castel, Robert. 1980. El orden psiquiátrico. Madrid, Ediciones La Piqueta.

Correa, Ana y colaboradores. 2003. Notas para una Psicología Social. Universidad Nacional de Córdoba.

Duschatzky, Silvia y Cristina Corea. 2002. Chicos en banda. Los caminos de la subjetividad en el declive de las instituciones. Buenos Aires, Paidós.

López, Ema y Enrique, José. 2004. "Del sujeto a la agencia (a través de lo político)". España. En Atenea digital. Nro. 5, Primavera,

Fernández, Ana y otros. 2006. Política y Subjetividad. Asambleas barriales y fábricas recuperadas. Buenos Aires, Tinta y Limón.

Fernández Ana. 2007. Las lógicas colectivas. Imaginarios, cuerpos y multiplicidades. Buenos Aires, Biblos.

Foucault, Michael. 1992. Microfisica del poder. España. Ediciones la Piqueta.

Iriart, Celia, Howard Waitzkin, Jaime Breilh, Alfredo Estrada y Emerson, Elías Merhy. 2002. "Medicina social Latinoamericana: aportes y desafios". En Revista Panamericana de la 
Salud Pública. vol.12 no.2. Temas de actualidad. Current topics.

Jelin, Elizabeth 1998. Pan y afectos. La transformación de las familias. Buenos Aires, Fondo de Cultura Económica.

Julien, Philippe. 1993. El Manto de Noé: ensayo sobre la paternidad. Buenos Aires, Alianza Estudios.

Lewkowicz, Ignacio y Pablo Sztulwark. 2003. Arquitectura plus de sentidos. Buenos Aires, Altamira.

Margulis, Mario. 2009. Sociología de la cultura: conceptos y problemas. Buenos Aires, Biblos.

Montero, Maritza. 2004. "Introducción a la Psicología Comunitaria”. Buenos Aires, Paidós.

Morin, Edgar. 1994 “Epistemología de la complejidad”, en Fried Schnitman, D (comp.) Nuevos paradigmas, cultura y subjetividad. Buenos Aires, Paidós.

Quiroga, Ana. 1996. Crisis, procesos sociales, sujeto y grupo: desarrollo en psicología social a partir del pensamiento de enrique Pichon-Riviere. Buenos Aires, Edición Cinco.

Reguillo Cruz, Rossana 2006 "Políticas de la mirada: hacia una antropología de las pasiones contemporáneas. En Duseel, Inés. Educar la mirada: políticas y pedagogías de la infancia. Buenos Aires, Manantial.

Reguillo Cruz, Rossana. 2000. La clandestina centralidad de la vida cotidiana. En la vida cotidiana y su espacio-temporalidad. Barcelona, Anthropos.

Samaja, Juan. 2004. Epistemología de la salud. Buenos Aires, Lugar.

Svampa, Maristella. 2008. Cambio de época: movimientos sociales y poder político. Buenos Aires, Siglo XXI. 